\title{
Two Temperature Heat Flux of Semi Infinite Piezoelectric Ceramic Rod
}

\author{
Essam Bassiouny ${ }^{1,2 *}$, Refaat Sabry ${ }^{1,3,4}$, Hamdy Youssef $^{5}$ \\ ${ }^{1}$ Faculty of Science and Humanitarian Studies, Salman Bin Abdulaziz University, Al-Kharj, KSA \\ ${ }^{2}$ Department of Mathematics, Faculty of Science, Fayoum University, Fayoum, Egypt \\ ${ }^{3}$ Theoretical Physics Group, Department of Physics, Faculty of Science, Mansoura University, \\ Damietta Branch, New Damietta, Egypt \\ ${ }^{4}$ International Centre for Advanced Studies in Physical Sciences, Faculty of Physics and Astronomy, \\ Ruhr University Bochum, Bochum, Germany \\ ${ }^{5}$ Mechanical Engineering Department, Faculty of Engineering and Islamic Architecture, \\ Umm Al-Qura University, Mecca, KSA \\ Email: "esambassiouny@yahoo.com, sabryphys@yahoo.com,youssefanne2005@gmail.com
}

Received November 14, 2012; revised February 18, 2013; accepted February 25, 2013

\begin{abstract}
The theory of two-temperature generalized thermoelasticity is used to solve the problem of heating a semi-infinite rod made of a piezoelectric ceramic material within the framework of generalized thermopiezoelasticity theory by supplying the rod a certain amount of heat uniformly distributed over a finite time period to the finite end of the rod. The Laplace transform formalism is used to solve the proposed model. Inverse Laplace transforms are computed numerically using a method based on Fourier expansion techniques. The physical parameters (i.e., conductive temperature, dynamical temperature, stress, strain, and displacement distributions) are investigated graphically.
\end{abstract}

Keywords: Two Temperatures; Generalized Thermoelasticity; Thermopiezoelasticity; Thermal Waves; Ceramics; Piezoelectric Materials-Heat Flux

\section{Introduction}

Piezoelastic ceramics have become now prominent and extensively used in many engineering applications especially in mechatronics, structronic systems, sensors, actuators and intelligent structures [1-3].

Piezoelectric ceramic have been extensively used in many engineering and industrial applications. So, the theory of generalized thermo-piezoelectricity has been the object of numerous investigations in the last decades or so, concerning both its theoretical foundations and the applications.

It is very well known now that in the classical coupled and uncoupled theories of thermoelasticity, the heat conduction equations are of diffusion type. This means that the heat wave will propagate with Infinite speed, which contradicts the physical observations.

Widespread attention to eliminate this paradox has been given to thermoelasticity theories which admit a finite speed for the propagation of thermal waves. Many authors have formulated generalized theories involve a hyperbolic-type heat equation and are referred to as gen-

\footnotetext{
"Corresponding author.
}

eralized thermoelasticity.

Among all these theories, there are two different famous theories of generalized thermoelasticity. The first theory is based on the modified Fourier's Law of heat conduction and allows for one relaxation time. This is the Lord and Schulman theory [4]. The second theory was developed by Green and Lindsay [5]. It modifies both the energy equation and the Duhamel-Neuman relation. It admits two relaxation times. In this work we solve the problem of heating of a semi-infinite piezoelectric rod by supplying a certain amount of heating uniformly distributed over a finite time period to the finite end. The rod is assumed to be at rest at zero temperature initially.

\section{Governing Equations}

In the absence of body force, free charge and inner heat sources, we consider generalized thermo-piezoelectric governing differential equations [6,7] as follows: Equations of motion:

$$
\sigma_{i j, j}=\rho \ddot{u}_{i} .
$$

Equation of entropy increment (in the absence of inner heat source): 


$$
q_{i, i}=-T_{0} \dot{\zeta},
$$

Stress-strain-temperature:

$$
\sigma_{i j}=c_{i j k l} e_{k l}-h_{k i j} D_{k}-\beta_{i j} \theta,
$$

Gauss equation and electric field relation:

$$
\begin{aligned}
& D_{i, i}=0 \\
& E=-v_{, i}, \\
& E_{i}=h_{i k l} e_{k l}+\tau_{i k} D_{k}-d_{i} \theta .
\end{aligned}
$$

Equation of entropy density:

$$
\zeta=\beta_{i j} e_{i j}+d_{i} D_{i}+c T
$$

Strain-displacement relations:

$$
e_{i j}=\frac{1}{2}\left(u_{i, j}+u_{j, i}\right) .
$$

Fourier's law for heat conduction

$$
q_{i}+A_{i j} \dot{q}_{j}=-k_{i j} \varphi_{, j}
$$

where $\varphi$ is the conductive temperature and it satisfies the relation

$$
\varphi-T=a \varphi_{, i i},
$$

in which $a>0$ is the two-temperature parameter and $k_{i j}$ are the components of thermal conductivity tensor.

In the above equations, a comma followed by a suffix denotes material derivatives and a superposed dot denotes the derivatives with respect to time

\section{One Dimension Formulation}

Consider a semi-infinite piezoelectric rod occupying the region $x \geq 0$. At the near end a uniform flow of heat is supplied to the rod during a finite period of time. We assume the following form for the displacement component [6]:

$$
u_{x}=u(x, t), u_{y}=u_{z}=0 .
$$

We consider the following forms of the linearized basic equations in one-dimensional formulation:

$$
\begin{aligned}
& (\lambda+2 \mu) \frac{\partial^{2} u}{\partial x^{2}}-\gamma \frac{\partial \theta}{\partial x}=\rho \frac{\partial^{2} u}{\partial t^{2}}, \\
& \sigma=(\lambda+2 \mu) \frac{\partial u}{\partial x}-\gamma \theta-\hbar D \\
& k \frac{\partial^{2} \varphi}{\partial x^{2}}=\left(\frac{\partial}{\partial t}+\tau_{o} \frac{\partial^{2}}{\partial t^{2}}\right)\left[\rho C_{E} \theta+\gamma T_{o} e\right] \\
& q(x, t)+\tau_{o} \dot{q}(x, t)=-k \frac{\partial \varphi(x, t)}{\partial x} \\
& \varphi-T=a \frac{\partial^{2} \varphi}{\partial x^{2}},
\end{aligned}
$$

$$
\begin{gathered}
e=\frac{\partial u}{\partial x}, \\
\frac{\partial D}{\partial x}=0, \\
E=-\frac{\partial v}{\partial x},
\end{gathered}
$$

where $\gamma=\alpha_{t}(3 \lambda+2 \mu), \quad \alpha_{t}$ is the coefficient of the linear thermal expansion, $k$ is the coefficient of thermal conductivity and $x$ is the coordinate taken along the rod.

It is convenient now to introduce the following dimensionless variables:

$$
\begin{aligned}
& u^{\prime}=c_{o} \eta u, t^{\prime}=c_{o}^{2} \eta t, \sigma^{\prime}=\frac{\sigma}{(\lambda+2 \mu)}, \\
& \theta^{\prime}=\frac{T-T_{o}}{T_{o}}, t_{o}^{\prime}=c_{o}^{2} \eta t_{o}, x^{\prime}=c_{o} \eta x \\
& \tau_{o}^{\prime}=c_{o}^{2} \eta \tau_{o}, \varphi^{\prime}=\frac{\varphi-T_{o}}{T_{o}}, q^{\prime}=\frac{q}{k c_{o} \eta T_{o}}, \\
& D^{\prime}=\frac{h}{\lambda+2 \mu} D, \eta=\frac{\rho C_{E}}{k}, c_{o}^{2}=\frac{\lambda+2 \mu}{\rho}
\end{aligned}
$$

From Gauss's law, since there is no free charge inside the piezoelectric rod we have

$$
\frac{\partial D}{\partial x}=0,
$$

which gives

$$
D=\text { const. }
$$

Substituting from (20) into Equations (12)-(19) and dropping the primes for convenience, we obtain the following set of non-dimensional equations [6]:

$$
\begin{aligned}
& \frac{\partial^{2} e}{\partial x^{2}}-\alpha \frac{\partial^{2} \theta}{\partial x^{2}}=\frac{\partial^{2} e}{\partial t^{2}}, \\
& \sigma=e-\alpha \theta-D, \\
& \frac{\partial^{2} \varphi}{\partial x^{2}}=\left(\frac{\partial}{\partial t}+\tau_{o} \frac{\partial^{2}}{\partial t^{2}}\right)(\theta+\varepsilon e) \\
& q(x, t)+\tau_{o} \dot{q}(x, t)=-\frac{\partial \varphi(x, t)}{\partial x}
\end{aligned}
$$

and the following relation between the conductive temperature and the thermo dynamical one:

$$
\theta=\varphi-\omega \frac{\partial^{2} \varphi}{\partial x^{2}}
$$

where $\alpha=\frac{\gamma T_{o}}{(\lambda+2 \mu)}, \varepsilon=\frac{\gamma}{\rho C_{E}}$ and $\omega=a c_{0}^{2} \eta^{2}$.

The rod is supplying a certain amount of heat uniformly distributed over a finite period of time to the near end and is traction free. The boundary conditions are: 


$$
-\frac{\partial \bar{\varphi}(x, s)}{\partial x}=F_{o} \delta_{t_{o}}(t)
$$

where $F_{o}$ is the heat flux intensity and $\delta_{t_{o}}(t)$ the well-known impulse function:

$$
\begin{aligned}
& \delta_{t_{o}}(t)= \begin{cases}\frac{1}{t_{o}} & 0<t \leq t_{o} \\
0 & t>t_{o}\end{cases} \\
& \varphi(\infty, t)=0, \quad e(\infty, t)=0,0<t<\infty \\
& \sigma(\infty, t)=0, \quad \sigma(0, t)=0, \quad 0<t<\infty
\end{aligned}
$$

while the initial conditions are assumed to be:

$$
\varphi(x, 0)=0, e(x, 0)=0, \sigma(x, 0)=0 \quad 0 \leq x \leq \infty
$$

Applying the Laplace transform defined by:

$$
L\{f(t)\}=\overline{f(s)}=\int_{0}^{\infty} \mathrm{e}^{-s t} f(t) \mathrm{d} t
$$

to both sides of Equations (23)-(27), we obtain:

$$
\begin{aligned}
& \frac{\mathrm{d}^{2} \bar{e}}{\mathrm{~d} x^{2}}-\alpha \frac{\mathrm{d}^{2} \bar{\theta}}{\mathrm{d} x^{2}}=s^{2} \bar{e}, \\
& \bar{\sigma}=\bar{e}-\alpha \bar{\theta}-\frac{D}{s}, \\
& \frac{\mathrm{d}^{2} \bar{\varphi}}{\mathrm{d} x^{2}}=\left(s+\tau_{o} s^{2}\right) \bar{\theta}+\varepsilon\left(s+\tau_{o} s^{2}\right) \bar{e}, \\
& \left(1+\tau_{o} s\right) \bar{q}(x, s)=-\frac{\partial \bar{\varphi}(x, s)}{\partial x}, \\
& \bar{\theta}=\bar{\varphi}-\omega \frac{\mathrm{d}^{2} \bar{\varphi}}{\mathrm{d} x^{2}},
\end{aligned}
$$

where $s$ denotes the complex argument related to the Laplace transform.

The transformed boundary conditions take the forms

$$
-\frac{\partial \bar{\varphi}(0, s)}{\partial x}=\frac{F_{o}\left(1-\mathrm{e}^{-s t_{o}}\right)}{t_{o} s}=\bar{\varphi}_{o}
$$

Thus, the transformed component of the heat flux vector becomes

$$
\bar{q}(0, s)=\frac{\bar{\varphi}_{o}}{\left(1+\tau_{o} s\right)}=\bar{q}_{o}
$$

while the Equations (30) become

$$
\begin{aligned}
& \bar{\varphi}(\infty, t)=0, \quad \bar{e}(\infty, t)=0, \\
& \bar{\sigma}(\infty, t)=0, \quad \bar{\sigma}(0, t)=0,
\end{aligned}
$$

and the corresponding transformed initial conditions of the Equations (31) assume the form:

$$
\bar{\varphi}(x, 0)=\bar{e}(x, 0)=\bar{\sigma}(x, 0)=0 \quad 0 \leq x \leq \infty
$$

Eliminating $\bar{\theta}$ between Equations (35) and (37), we get:

$$
\frac{\mathrm{d}^{2} \bar{\varphi}}{\mathrm{d} x^{2}}=L \bar{\varphi}+L \varepsilon \bar{e}
$$

where $L=L(s)=\frac{s+\tau_{0} s^{2}}{1+\omega\left(s+\tau_{0} s^{2}\right)}$.

Substituting from Equation (42) into Equation (37), we obtain

$$
\bar{\theta}=(1-\omega L) \bar{\varphi}-\omega L \varepsilon \bar{e} .
$$

Using Equation (42) we can easily eliminate $\bar{\theta}$ between Equations (33) and (44) to obtain

$$
\frac{\mathrm{d}^{2} \bar{e}}{\mathrm{~d} x^{2}}=M \bar{\varphi}+N \bar{e},
$$

where

$$
\begin{aligned}
& M=M(s)=\frac{\alpha L(1-\omega L)}{1+\omega \alpha \varepsilon L} \\
& N=N(s)=\frac{s^{2}+\alpha \varepsilon L(1-\omega L)}{1+\omega \alpha \varepsilon L}
\end{aligned}
$$

Solving Equations (42) and (45) together we get the following fourth order equation

$$
\left[k^{4}-a k^{2}+b\right] \bar{\varphi}=0
$$

where, $a=L+N$ and $b=L N-L \varepsilon M$. It is worth mentioning here that the roots of Equation (47) are functions of $s$ and assume the forms:

$$
k_{1}= \pm \frac{\sqrt{a+\sqrt{a^{2}-4 b}}}{\sqrt{2}}, k_{2}= \pm \frac{\sqrt{a-\sqrt{a^{2}-4 b}}}{\sqrt{2}},
$$

where

$$
\begin{aligned}
& a=\frac{s\left(1+\alpha \varepsilon+s\left(1+\tau_{o}+\alpha \varepsilon \tau_{o}+s\left(1+s \tau_{o}\right) \omega\right)\right)}{\left(1+s(1+\alpha \varepsilon)\left(1+s \tau_{o}\right) \omega\right)} \\
& b=\frac{s^{3}\left(1+s \tau_{o}\right)}{\left(1+s(1+\alpha \varepsilon)\left(1+s \tau_{o}\right) \omega\right)}
\end{aligned}
$$

Thus the solutions of the Equations (42) and (45) satisfying the boundary conditions at infinity are:

$$
\begin{aligned}
& \bar{\varphi}=A \mathrm{e}^{-x k_{1}}+B \mathrm{e}^{-x k_{2}} \\
& \bar{e}=C \mathrm{e}^{-x k_{1}}+D \mathrm{e}^{-x k_{2}}
\end{aligned}
$$

respectively, where $k_{1}, k_{2}$ are the roots of the Equation (47) and $A, B$ are parameters depending on $s$ to be determined using the boundary conditions while the relations between the coefficients $A, B, C, D$ are: 


$$
C=\frac{\left(K_{1}^{2}-L\right) A}{L \varepsilon}, \quad D=\frac{\left(K_{2}^{2}-L\right) B}{L \varepsilon}
$$

Using the boundary conditions (38) and (40) it is easy to verify that:

$$
A=-\frac{\left(k_{2}^{2}-L\right) \bar{\varphi}_{o}}{G}, \quad B=\frac{\left(k_{1}^{2}-L\right) \bar{\varphi}_{o}}{G},
$$

where

$$
G=k_{2}\left(k_{1}^{2}-L\right)-k_{1}\left(k_{2}^{2}-L\right)
$$

Substituting form Equations (54) into the Equation (53) we can easily evaluate the coefficients $C$ and $D$ :

$$
C=-D=-\frac{\left(k_{1}^{2}-L\right)\left(k_{2}^{2}-L\right) \bar{\varphi}_{o}}{L \varepsilon G},
$$

Therefore, the transformed heat conduction and the strain given by the Equations (51) and (52) respectively become:

$$
\bar{\varphi}=\bar{\varphi}_{o}\left(\varphi_{1} \mathrm{e}^{-x k_{2}}-\varphi_{2} \mathrm{e}^{-x k_{1}}\right)
$$

where

$$
\varphi_{1}=\frac{\left(k_{1}^{2}-L\right)}{G}, \varphi_{2}=\frac{\left(k_{2}^{2}-L\right)}{G},
$$

and

$$
\bar{e}=e_{1} \bar{\varphi}_{o}\left[\mathrm{e}^{-x k_{2}}-\mathrm{e}^{-x k_{1}}\right]
$$

where

$$
e_{1}=\frac{\left(k_{1}^{2}-L\right)\left(k_{2}^{2}-L\right) \bar{\varphi}_{o}}{L \varepsilon G} .
$$

Substituting the expressions of $\bar{\varphi}$ and of $\bar{e}$ from Equations (57) and (59) into the Equation (44) we get the following form of the thermodynamical function $\bar{\theta}$ :

$$
\bar{\theta}=\frac{\bar{\varphi}_{o}}{G}\left[\theta_{1} \mathrm{e}^{-x k_{2}}+\theta_{2} \mathrm{e}^{-x k_{1}}\right],
$$

where

$$
\theta_{1}=\left(1-\omega k_{2}^{2}\right)\left(k_{1}^{2}-L\right), \quad \theta_{2}=\left(1-\omega k_{1}^{2}\right)\left(k_{2}^{2}-L\right),
$$

Using Equations (59) and (61) it is easy to put $\bar{\sigma}$ given by the Equation (34) in the form:

$$
\begin{aligned}
\bar{\sigma} & =\frac{\bar{\varphi}_{o}}{L \varepsilon G}\left(\sigma_{1} \mathrm{e}^{-x k_{2}}-\sigma_{2} \mathrm{e}^{-x k_{1}}\right)-\frac{D}{s} \\
\sigma_{1} & =\left[\left(k_{2}^{2}-L\right)\left(k_{1}^{2}-L\right)-\alpha L \varepsilon \theta_{1}\right], \\
\sigma_{2} & =\left[\left(k_{1}^{2}-L\right)\left(k_{2}^{2}-L\right)-\alpha L \varepsilon \theta_{2}\right]
\end{aligned}
$$

Equations (57), (59), (61) and (63) are the complete solutions of the $\bar{\varphi}, \bar{e}, \bar{\theta}$ and $\bar{\sigma}$ respectively in the Laplace transformed domain.
In order to invert the Laplace transform, we adopt a numerical inversion method based on a Fourier series expansion [8].

Using this method, the inverse $f(t)$ of the Laplace transform $\bar{f}(s)$ is approximated by

$$
\begin{gathered}
f(t)=\frac{\mathrm{e}^{c t}}{t_{1}}\left[\frac{1}{2} \bar{f}(c)+\operatorname{Re} \sum_{k=1}^{N} \bar{f}\left(c+\frac{\mathrm{i} k \pi}{t_{1}}\right) \exp \left(\frac{\mathrm{i} k \pi t}{t_{1}}\right)\right] \\
0<t_{1}<2 t,
\end{gathered}
$$

where $N$ is a sufficiently large integer representing the number of terms in the truncated Fourier series, chosen such that

$$
\exp (c t) \operatorname{Re}\left[\bar{f}\left(c+\frac{N \pi}{t_{1}}\right) \exp \left(\frac{\mathrm{i} N \pi t}{t_{1}}\right)\right] \leq \varepsilon_{1},
$$

where $\varepsilon_{1}$ is a prescribed small positive number that corresponds to the degree of accuracy required and Re is the real part. The parameter $c$ is a positive free parameter that must be greater than the real part of all the singularities of $\bar{f}(s)$. The optimal choice of $c$ was obtained according to the criteria described in

\section{Numerical Results and Discussion}

Distributions of unknown functions along the rod for three different time moments, $t=0.1,0.2$ and 0.3 and for different values of the relaxation time: $\tau_{o}=0.1$, $\tau_{o}=0.05$ and are obtained numerically where the effects of the presence of the two temperature parameter on the unknown functions are illustrated in Figures 1-28.

Figures 1-4 illustrate the effects of the presence of the two temperature parameter on the conductive temperature $\varphi$, thermodynamical temperature $\theta$, stress $\sigma$ and strain $e$ distribution curves. We note that the two temperature parameter has significant effects on the unknown function fields especially on the strain distribution and the conductive temperature at the nearest end of the rod, it increases the amplitude of the unknowns at the nearest end of the rod and it has no effects far from the nearest end as seen on Figures 1 and $\mathbf{4}$.

The presence of the two temperature parameter leads to removing decreases the number of peaks as shown in Figure 4.

Figures 5-13, illustrate the effects of the relaxation time $\left(\tau_{o}=0.05, \tau_{o}=0.1\right)$ in the presence and in the absent of the two temperature parameter. The distributions of all the unknown fields $\varphi, \theta, \sigma$ and $e$ are affected slightly according to the changes in the relaxation time.

The removed peaks in Figures $\mathbf{1 1}$ and $\mathbf{1 2}$ of the strain distribution due to the presence of the two temperature parameter.

Figures 13-20, illustrate the effects of change in time 


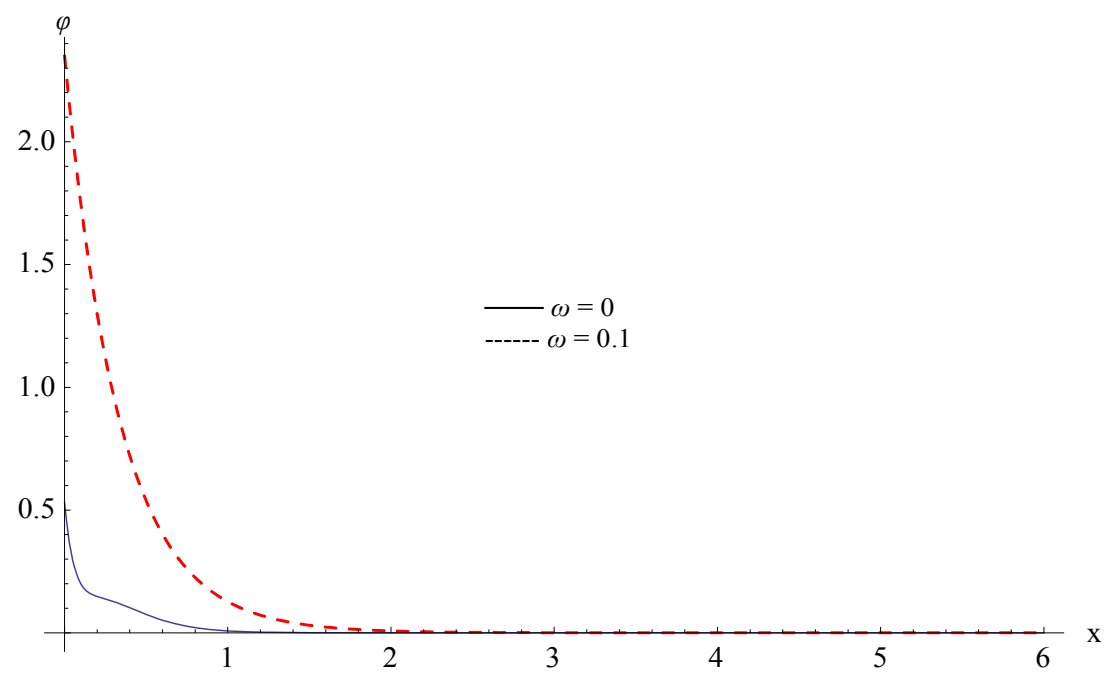

Figure 1. The effect of the two temperature parameter on the conductive heat distribution at $t_{o}=0.1, \tau_{o}=0.1$.

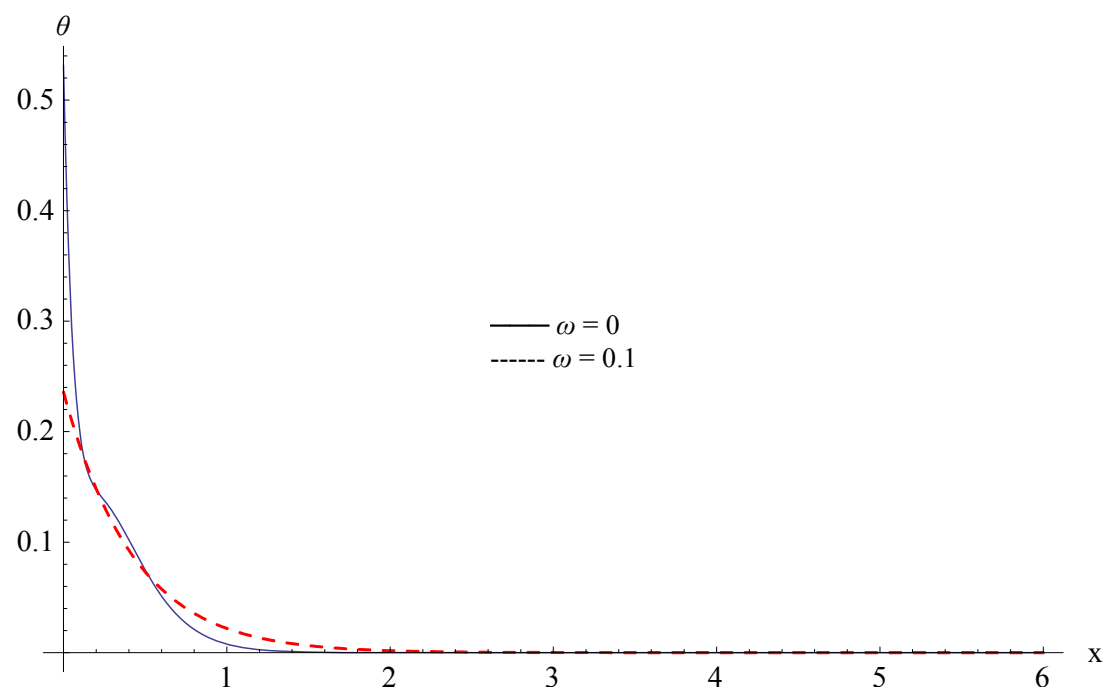

Figure 2. The effect of the two temperature parameter on the thermodynamical temperature at $t_{o}=0.1, \tau_{o}=0.1$.

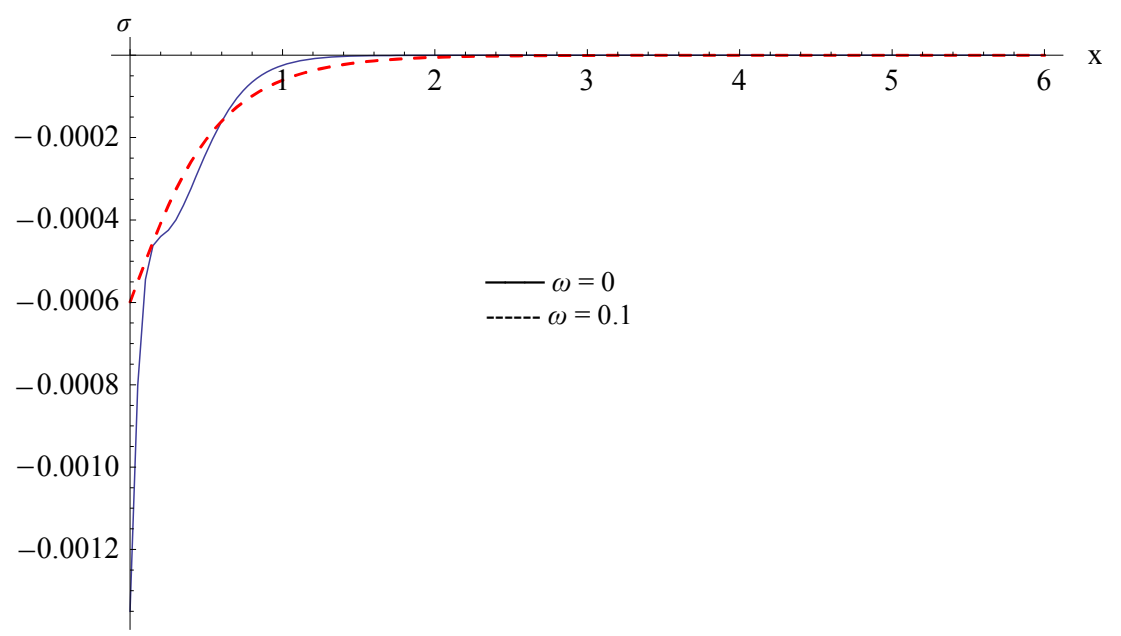

Figure 3. The effect of the two temperature parameter on the stress distribution at $t_{o}=0.1, \tau_{o}=0.1$. 


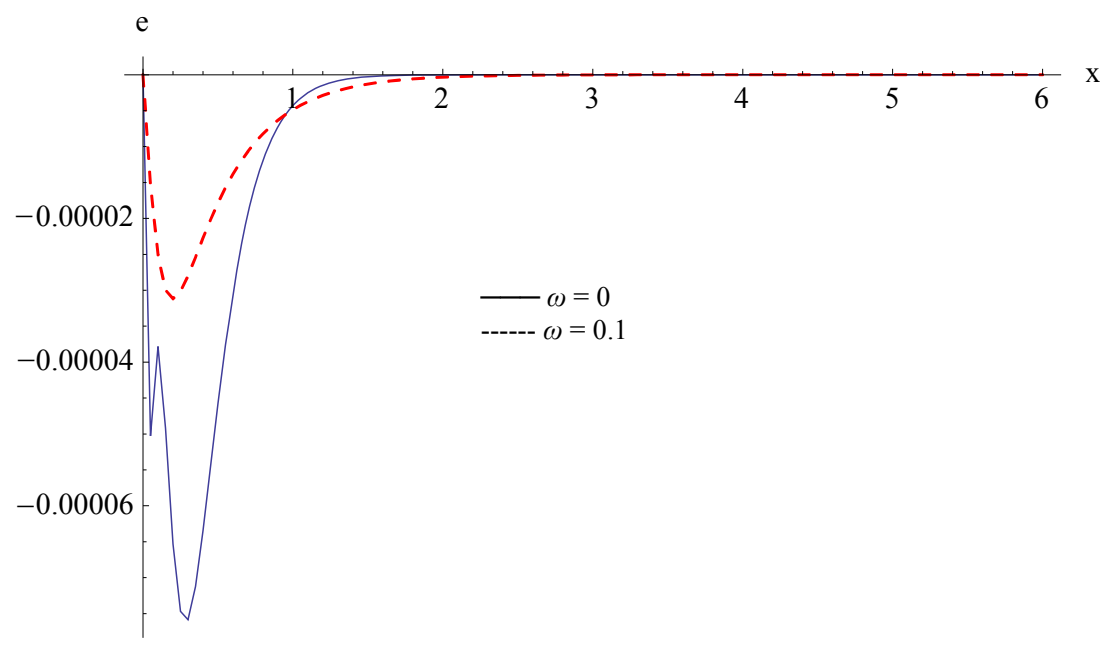

Figure 4. The effect of the two temperature parameteron the strain distribution at $t_{o}=0.1, \tau_{o}=0.1$.

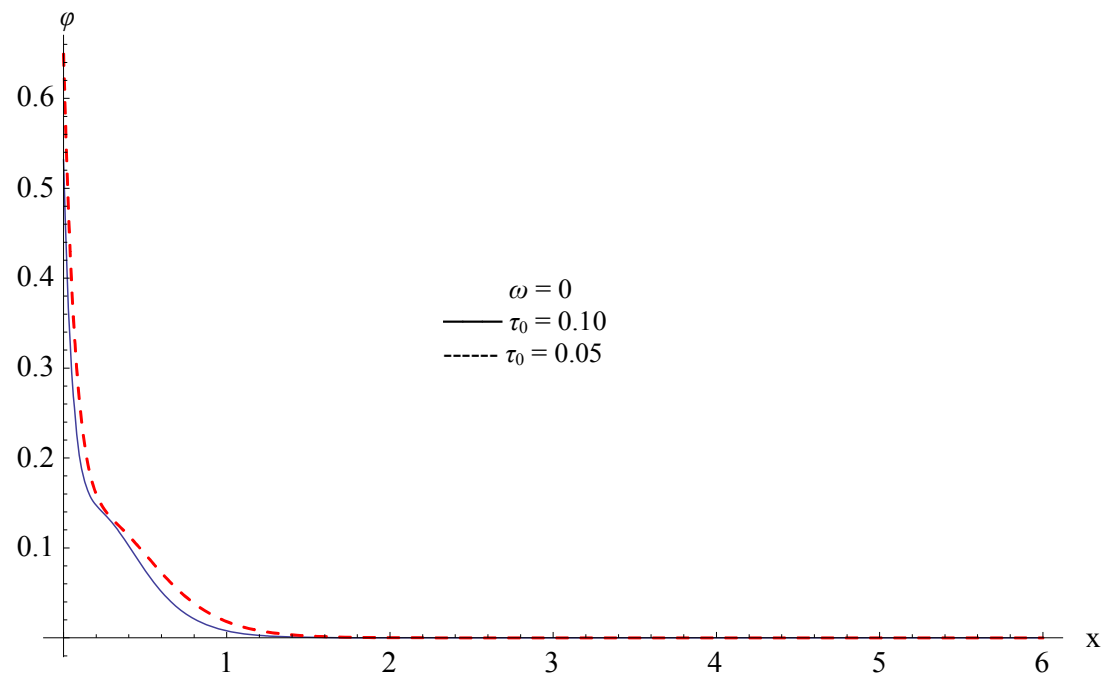

Figure 5. The effect of relaxation time on the heat conductive distribution in the absent of the two temperature parameter.

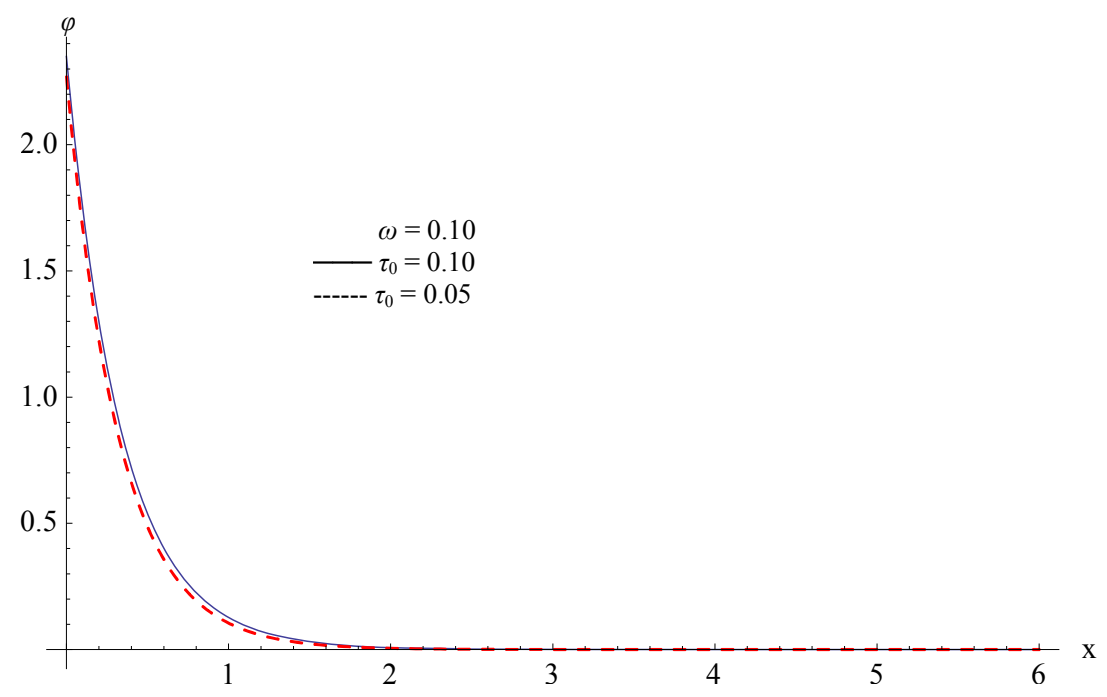

Figure 6. The effect of the two temperature parameter at different relaxation time on the heat conductive distribution. 


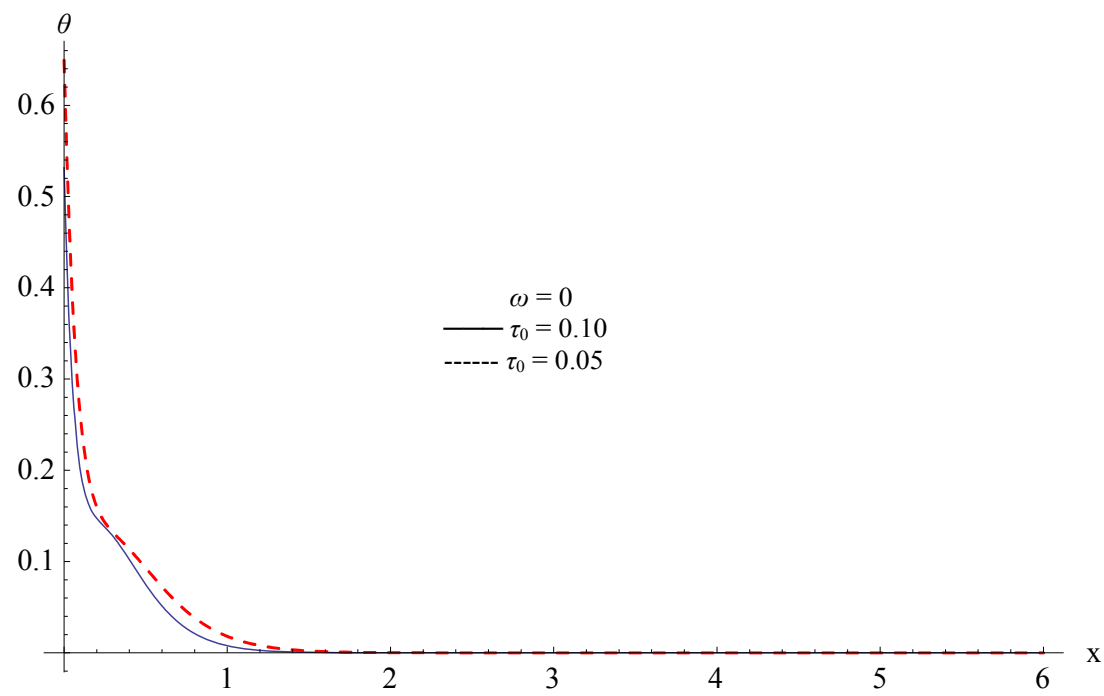

Figure 7. The effect of relaxation time on the thermodynamical temperature distribution in the absent of the two temperature parameter.

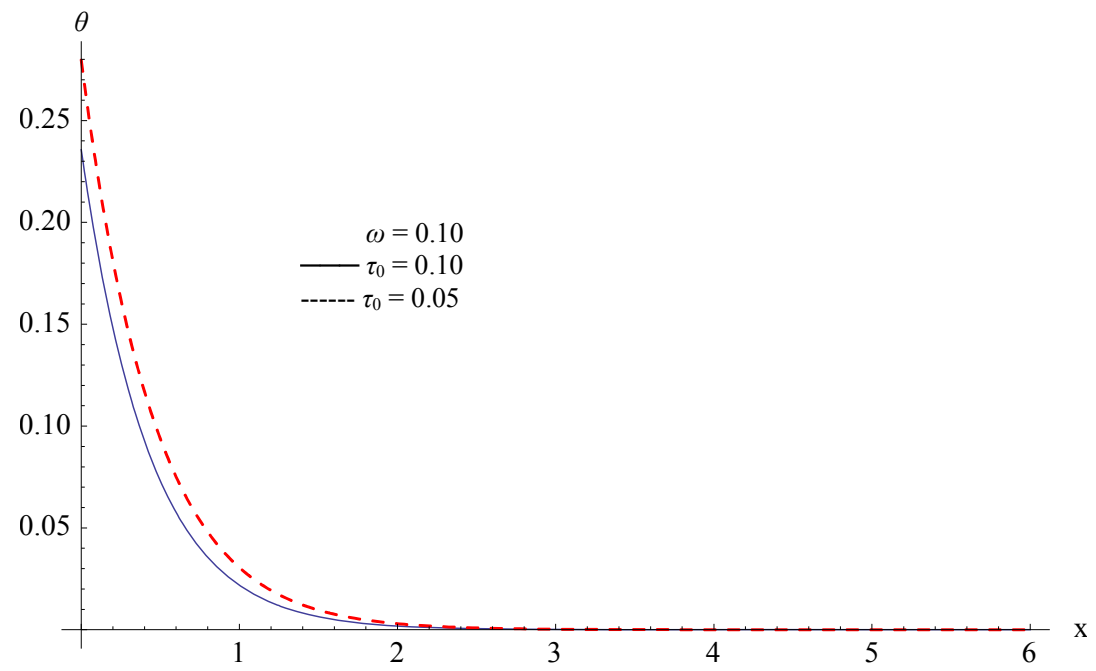

Figure 8. The effect of the two temperature parameter at different relaxation time on the thermodynamical temperature distribution.

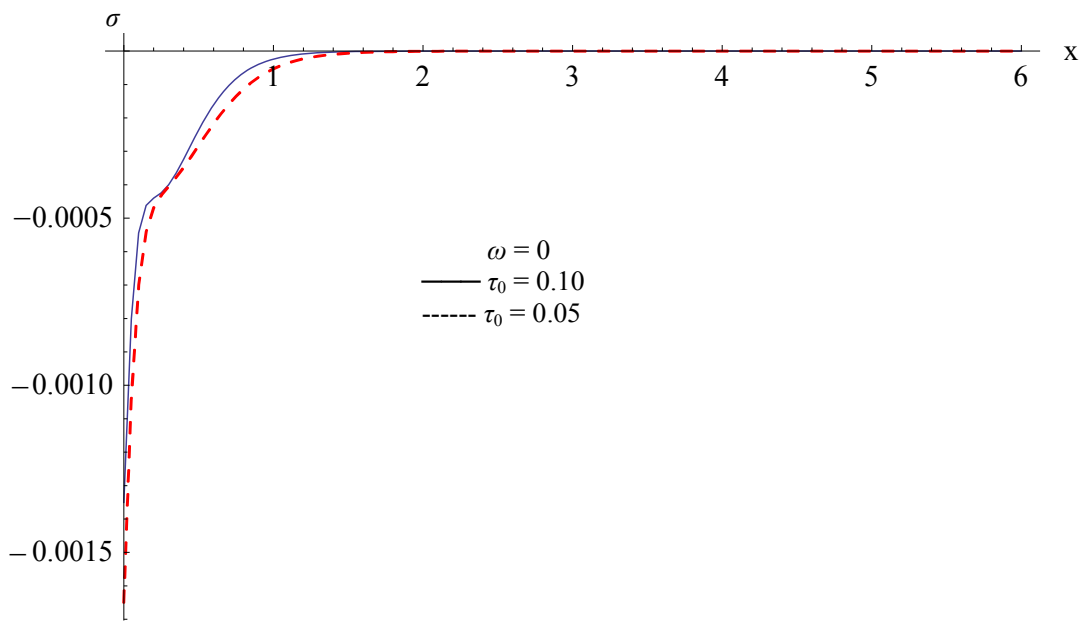

Figure 9. The effect of relaxation time on the stress distribution in the absent of the two temperature parameter. 


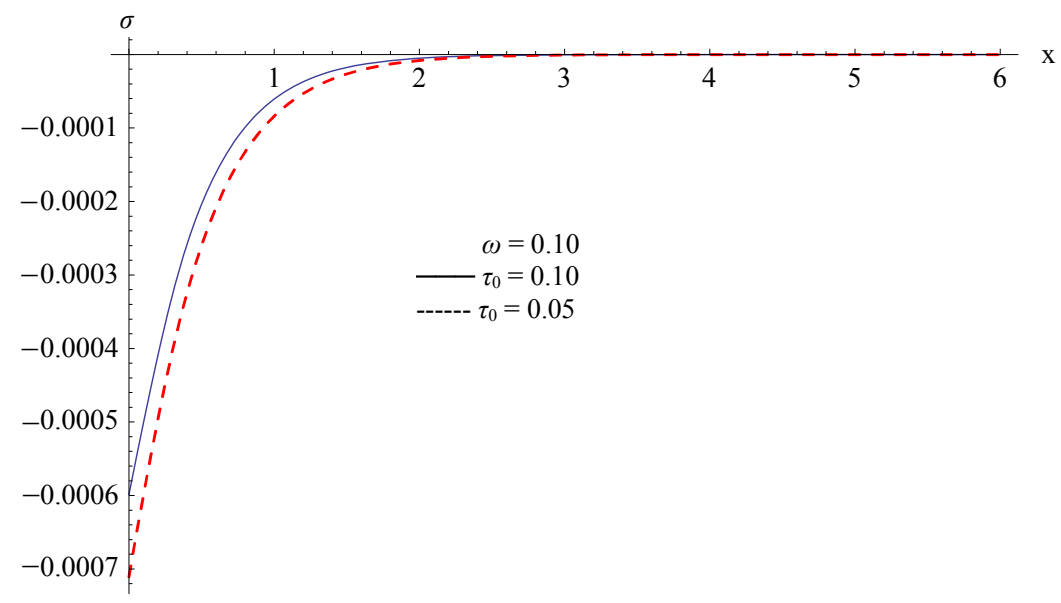

Figure 10. The effect of the two temperature parameter at different relaxation time on the stress distribution.

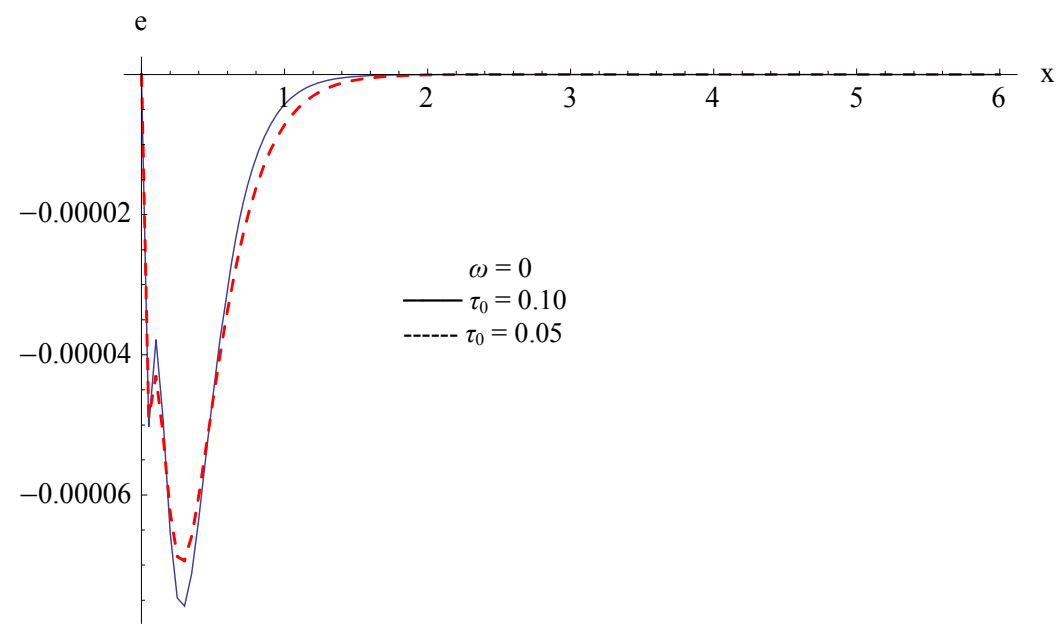

Figure 11. The effect of relaxation time on the strain distribution in the absent of the two temperature parameter.

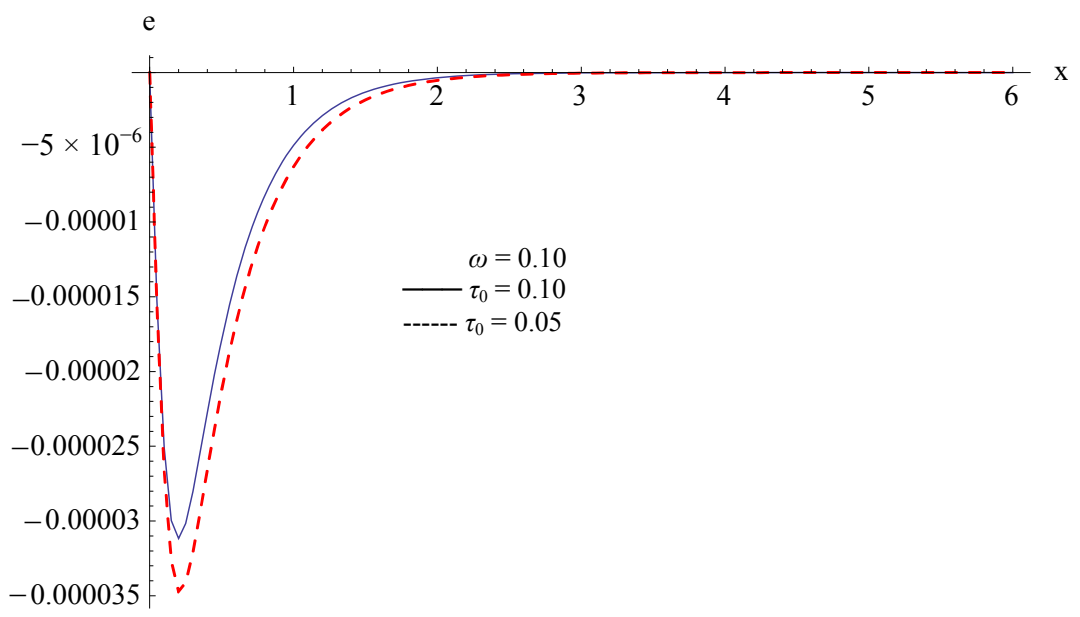

Figure 12. The effect of the two temperature parameter at different relaxation time on the strain distribution.

on the unknowns field $\varphi, \theta, \sigma$ and $e$. At different time the distribution of the conductive temperature suffer great changes especially in the absence of the two temperature parameter. The amplitude increases with time in the thermodynamical temperature (Figures 15 and 16) as well as the stress distribution curves (Figures 17 and 18) at different values of time. The effect of the two temperature parameter vanishes far from the nearest end of the rod. 


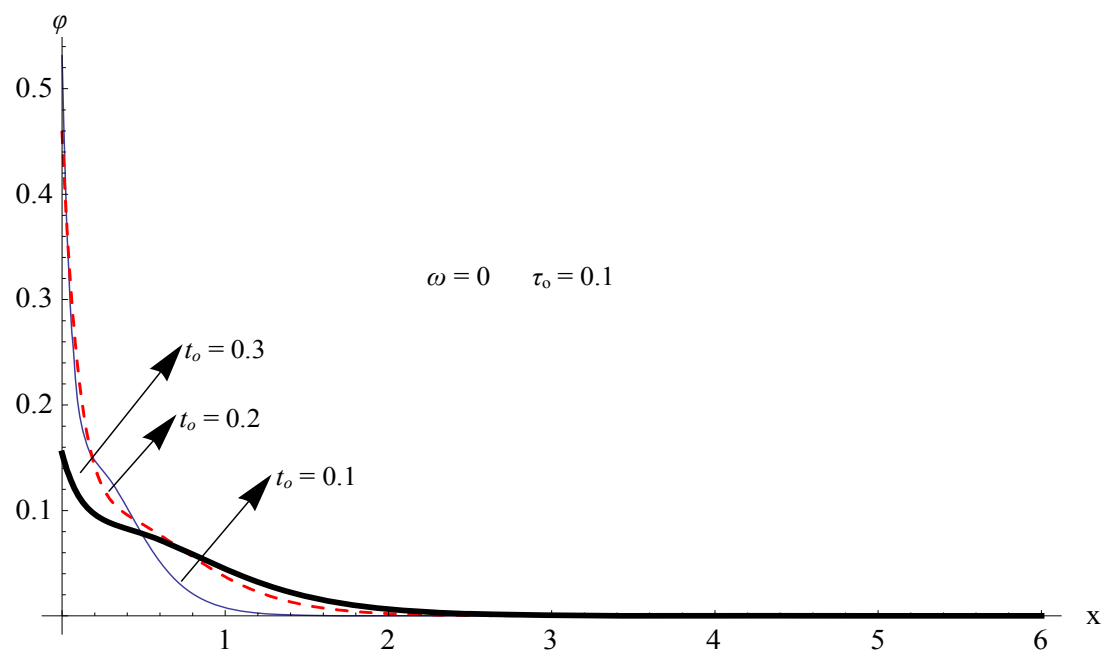

Figure 13. The heat conduction distribution at different time in the absent of two temperature parameter at $\tau_{o}=0.1$.

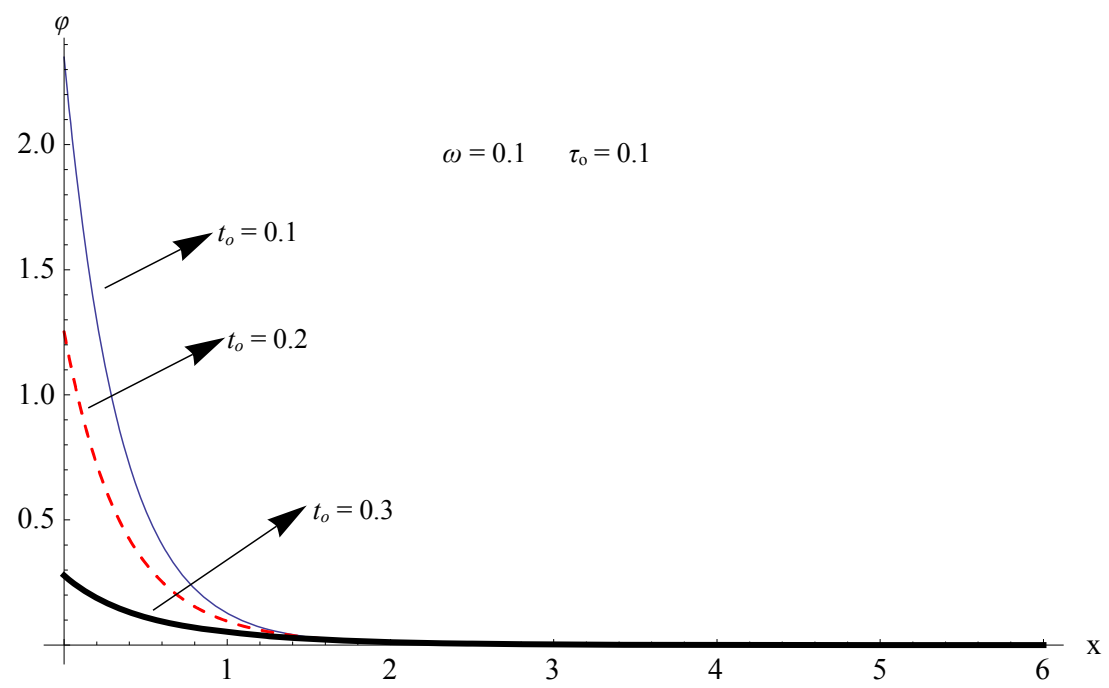

Figure 14. The heat conduction distribution at different time in the presence of two temperature parameter at $\tau_{o}=0.1$.

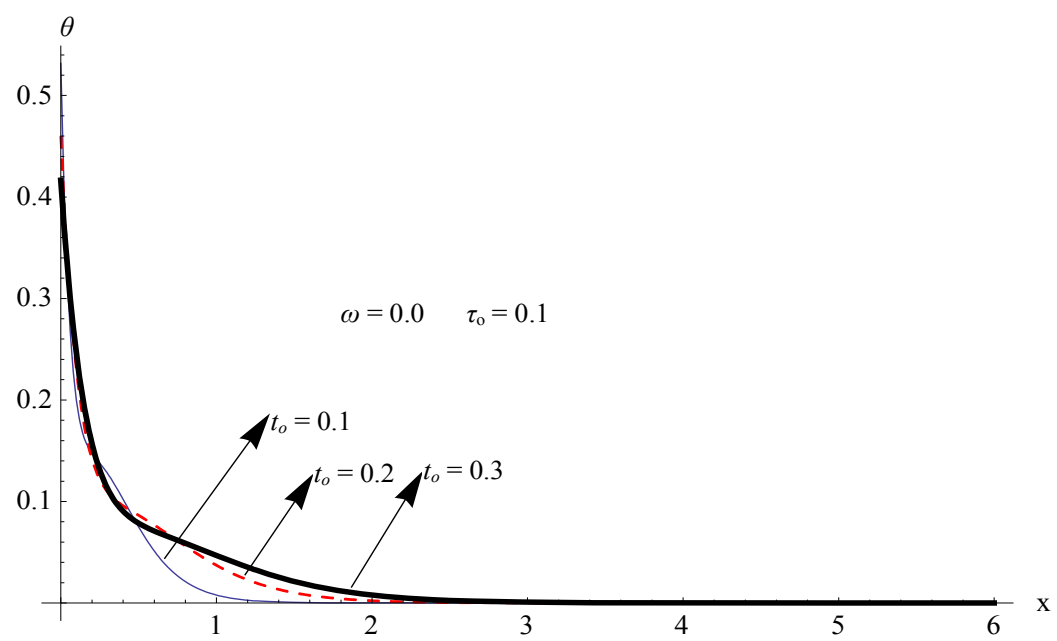

Figure 15. The thermodynamical temperature distribution at different time in the absent of two temperature parameter at $\tau_{o}=0.1$. 


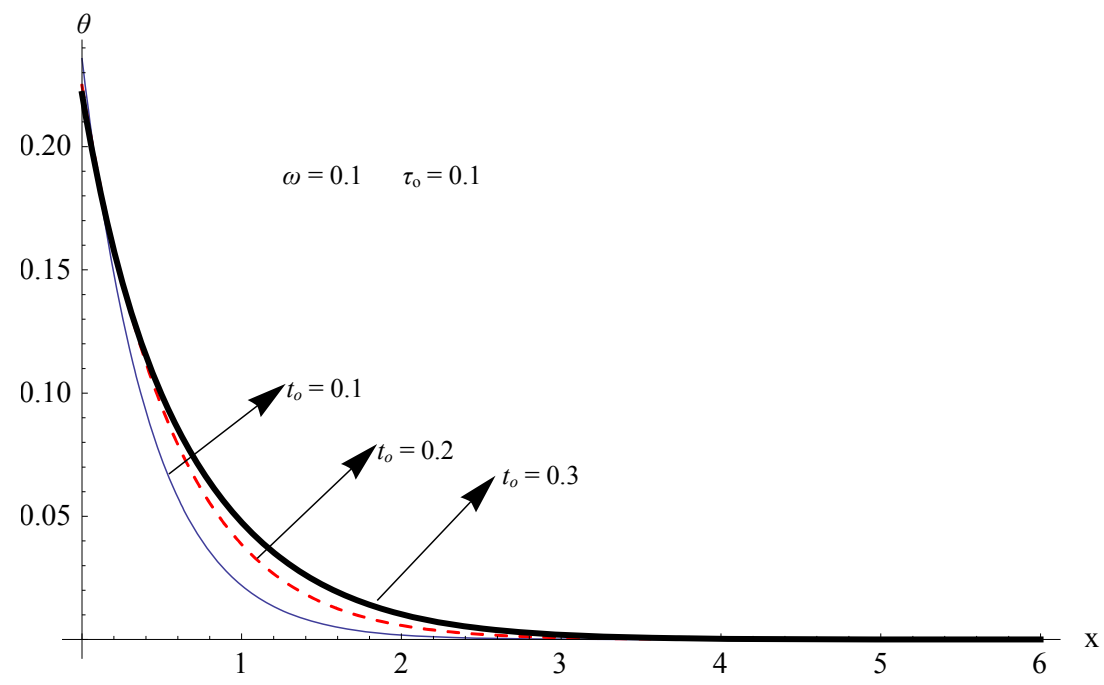

Figure 16. The thermodynamical temperature distribution at different time in the presence of two temperature parameter at $\tau_{o}=0.1$.

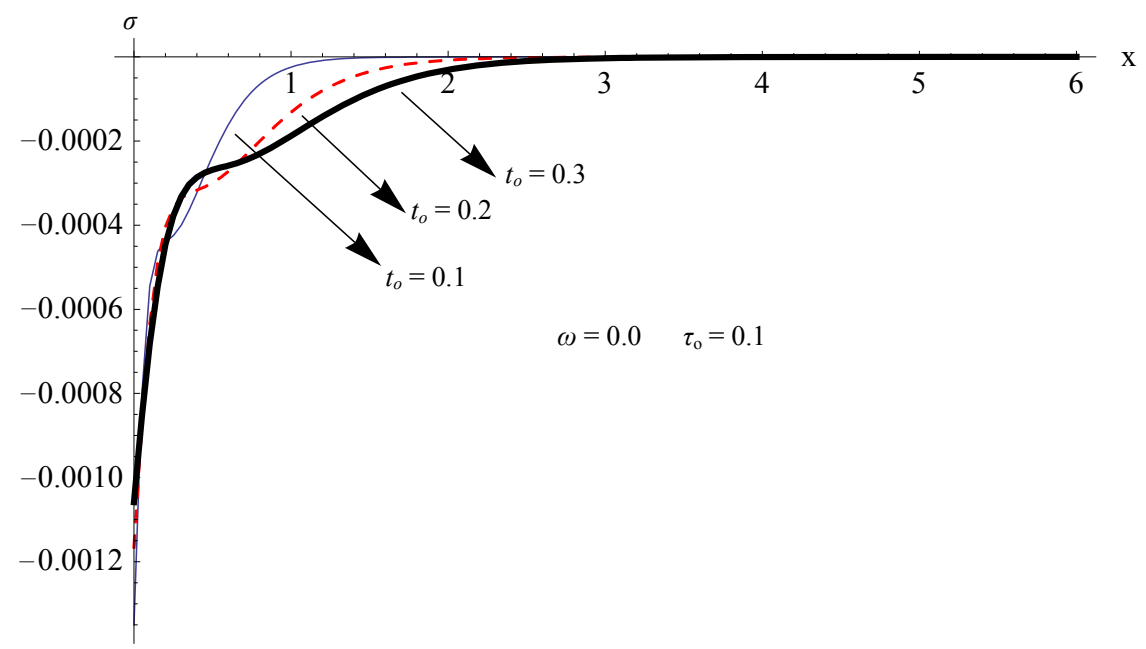

Figure 17. The stress distribution at different time in the absent of two temperature parameter at $\tau_{o}=0.1$.

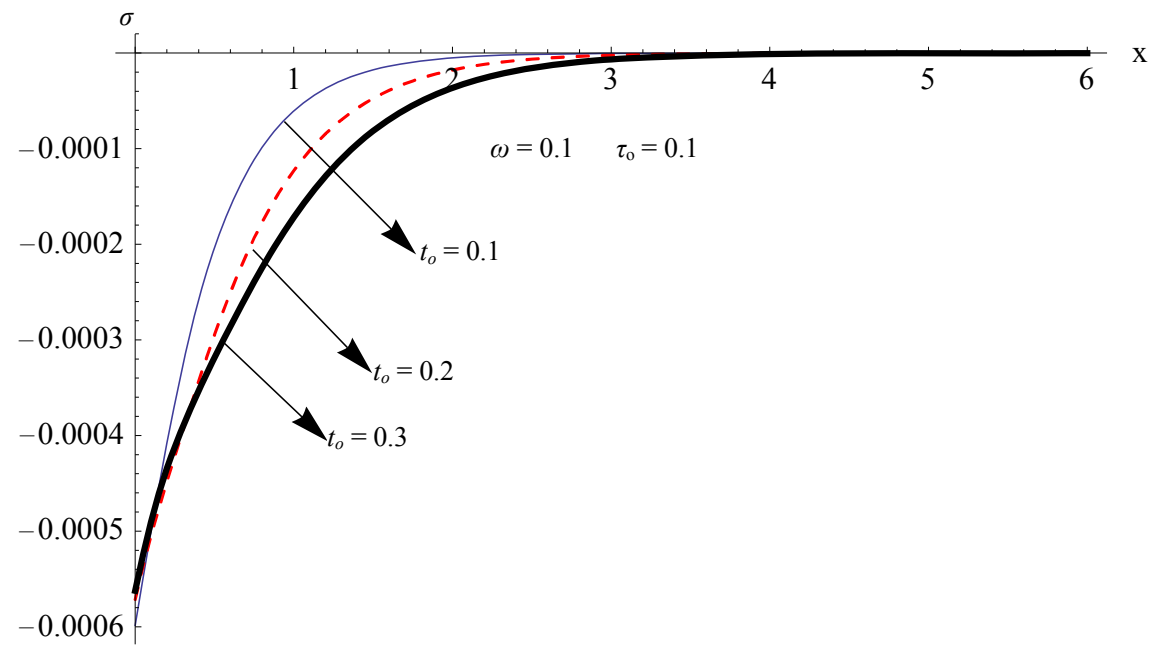

Figure 18. The stress distribution at different time in the presence of two temperature parameter at $\tau_{o}=0.1$. 


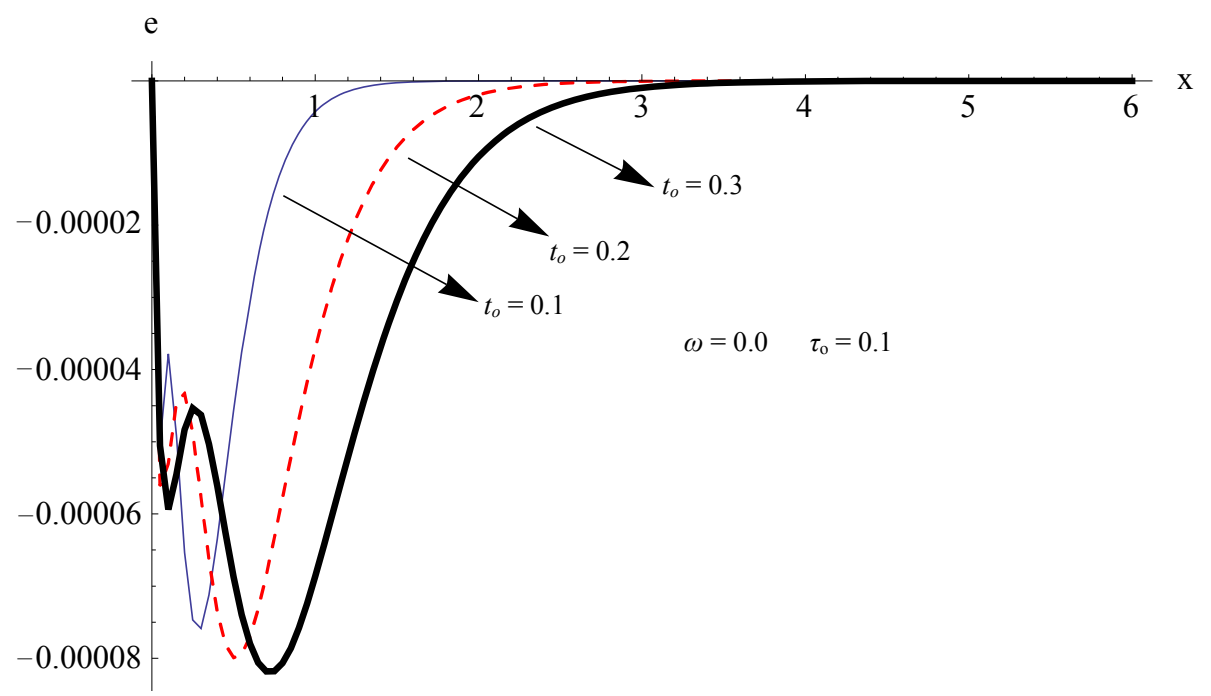

Figure 19. The strain distribution at different time in the absent of two temperature parameter $\tau_{o}=0.1$.

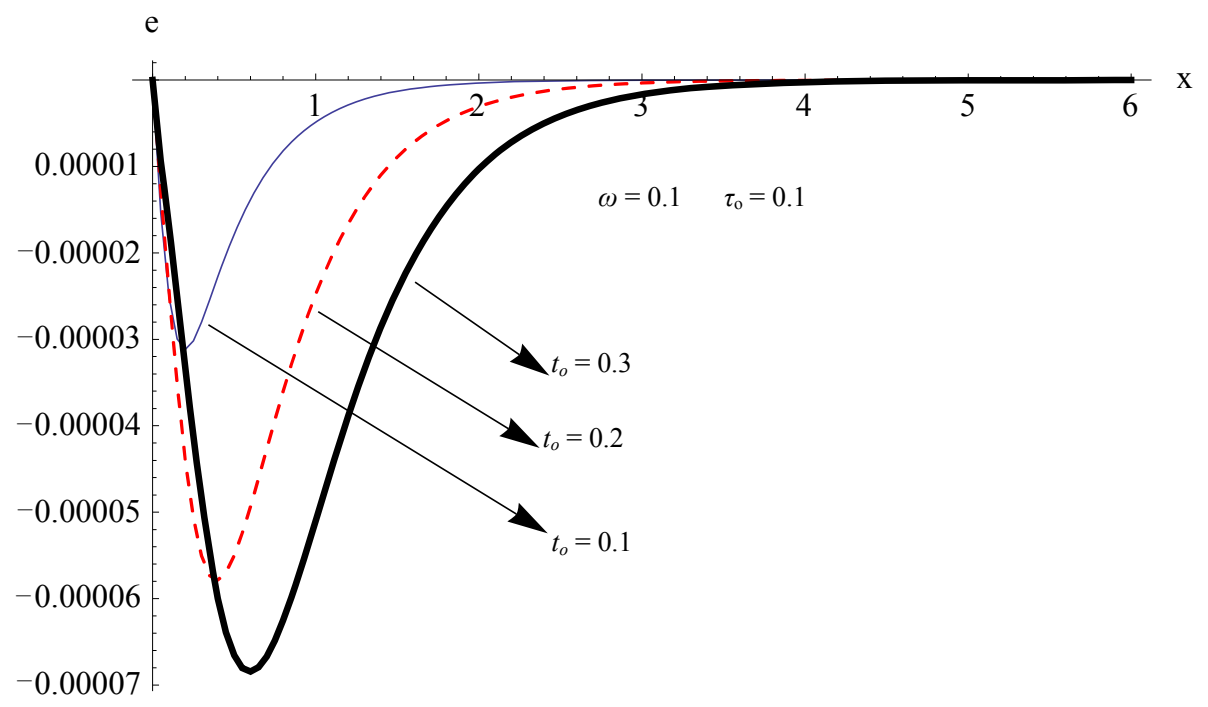

Figure 20. The strain distribution at different time in the presence of two temperature parameter at $\tau_{o}=0.1$.

Figures 19 and 20 indicate that as time increases, the changes in the strain distribution curves increases too. The curves of the strain in Figure 20 are smoother than that of figure 19 due to the presence of the two temperature parameter. In these figures the amplitude increases with time.

Figures 21-27 illustrate the changes in the distribution curves of the unknown fields. In this figures comparisons with the cases where the relaxation time $\tau_{o}=0.1$ shows that a slight effects of the change of the relaxation time on the unknown fields.

\section{Conclusion}

We considered a two temperature generalized thermppiezoelectric model to study the effects of different pa- rameters on the thermomechanical behavior of a semi infinite rod made of piezoelectric material. We can conclude that the presence of the two temperature parameter leads to a direct relation between the time and the amplitude of the thermodynamical temperature and an inverse relation with the heat conduction. The absolute values of the amplitude of the stress and the strain are in direct relation with the time for all values of the two temperature parameter. The values of the stress and the strain resemble the same sign for all values of time and the two temperature parameter.

\section{Acknowledgements}

The authors would like to thank the support of the Deanship of Scientific Research in Salman Bin Abdul-Aziz 


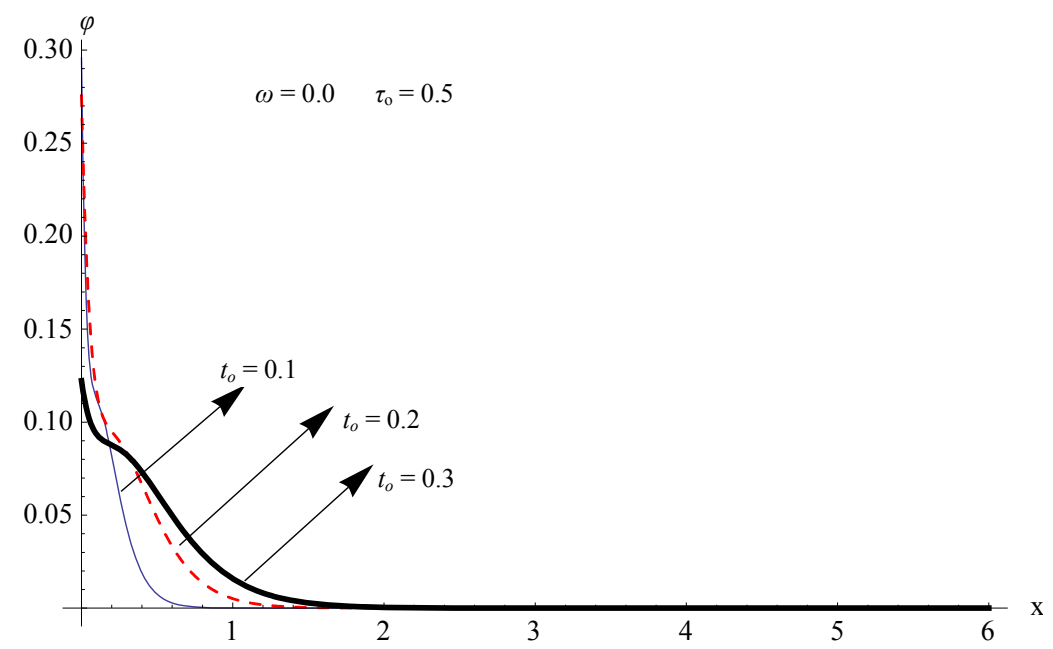

Figure 21. The heat conduction distribution at different time in the absent of two temperature parameter at $\tau_{o}=0.5$.

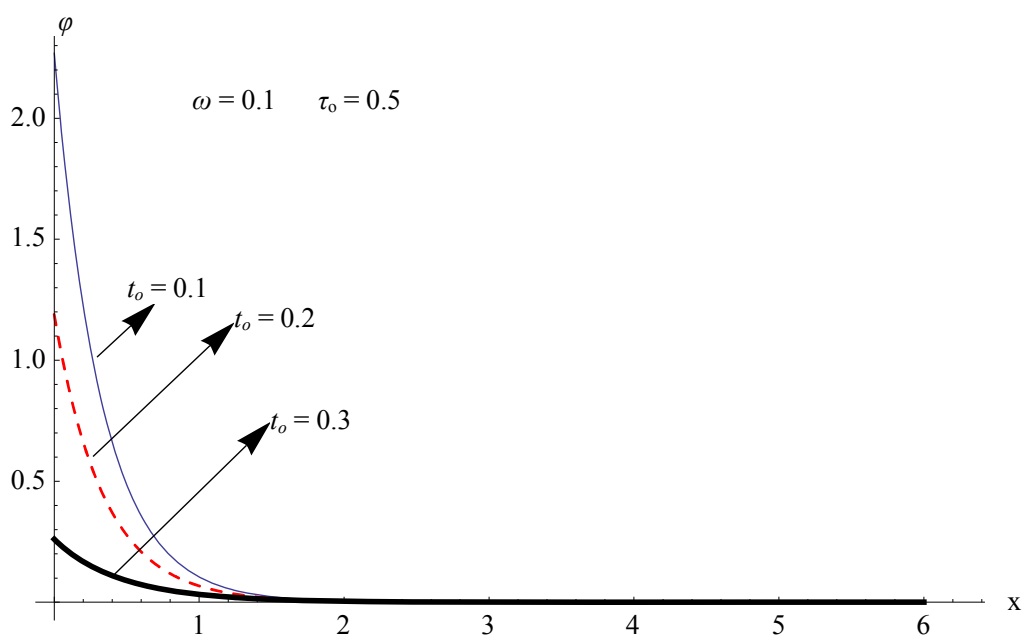

Figure 22. The heat conduction distribution at different time in the presence of two temperature parameter a $\tau_{o}=0.5$.

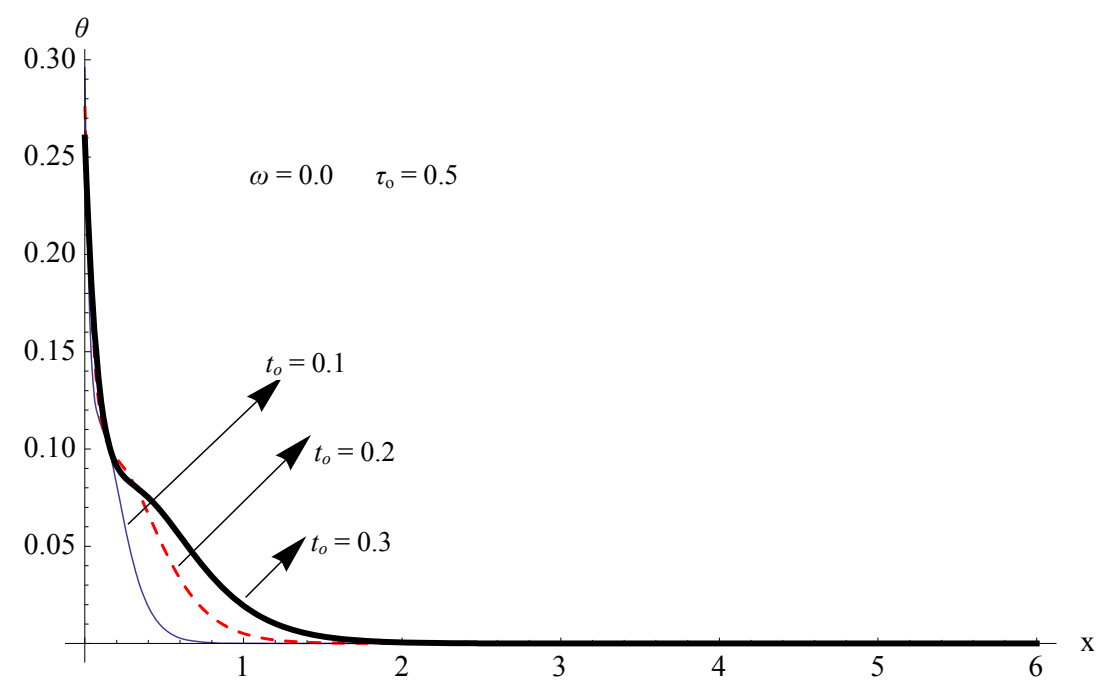

Figure 23. The thermodynamical temperature distribution at different time in the absent of two temperature parameter at $\tau_{o}=0.5$. 


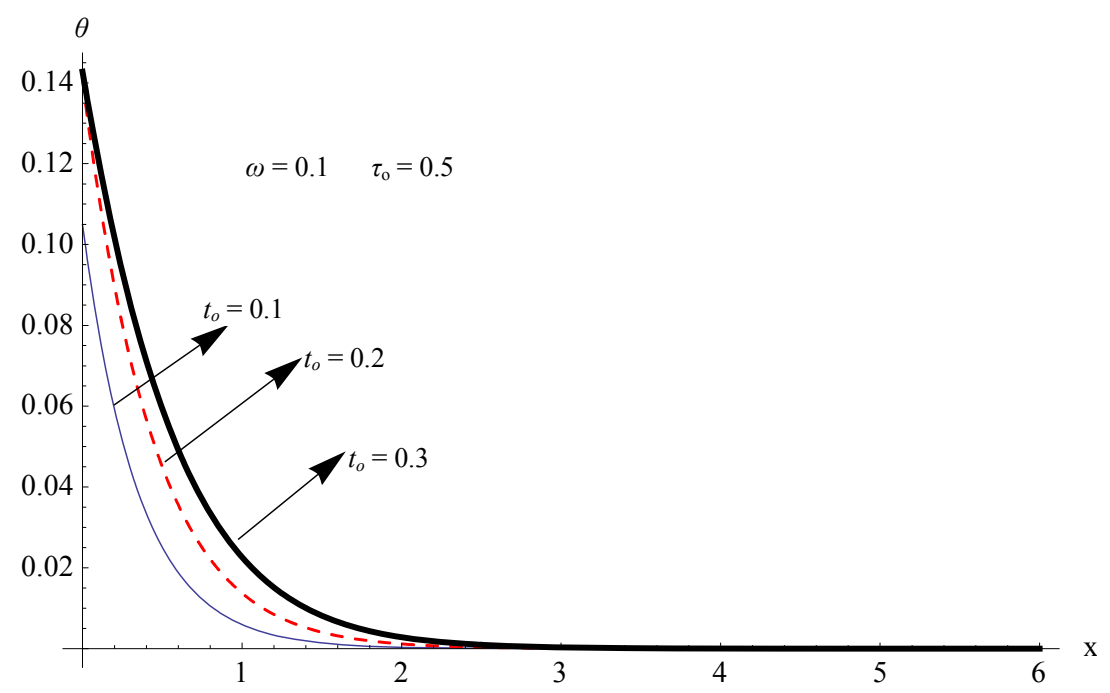

Figure 24. The thermodynamical temperature distribution at different time in the presence of two temperature parameter at $\tau_{o}=0.5$.

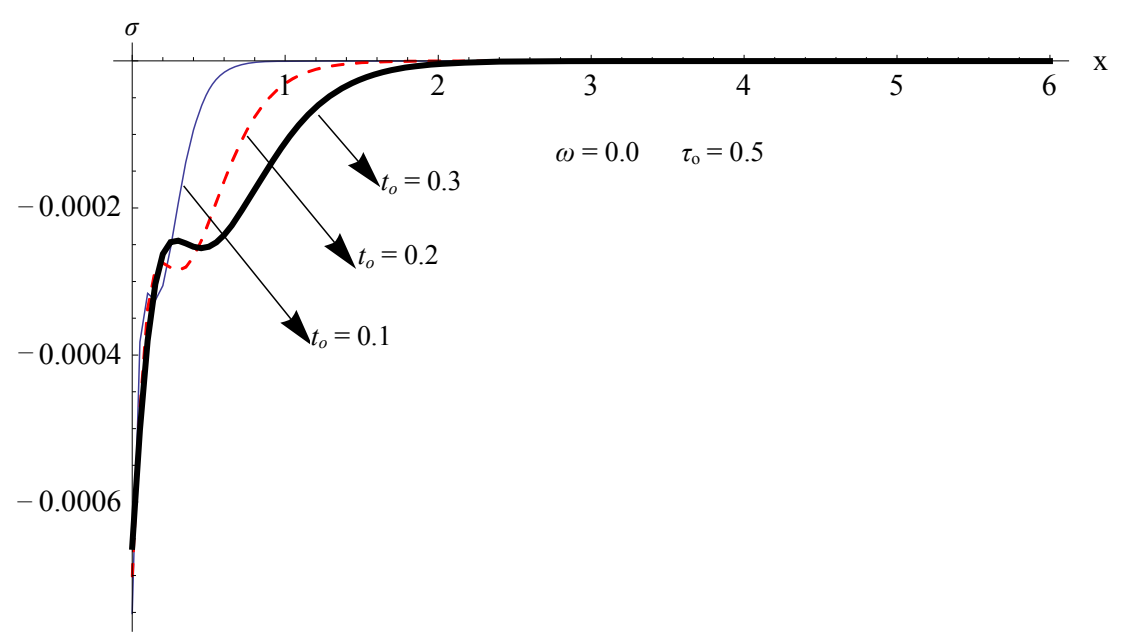

Figure 25. The stress distribution at different time in the absent of two temperature parameter at $\tau_{o}=0.5$.

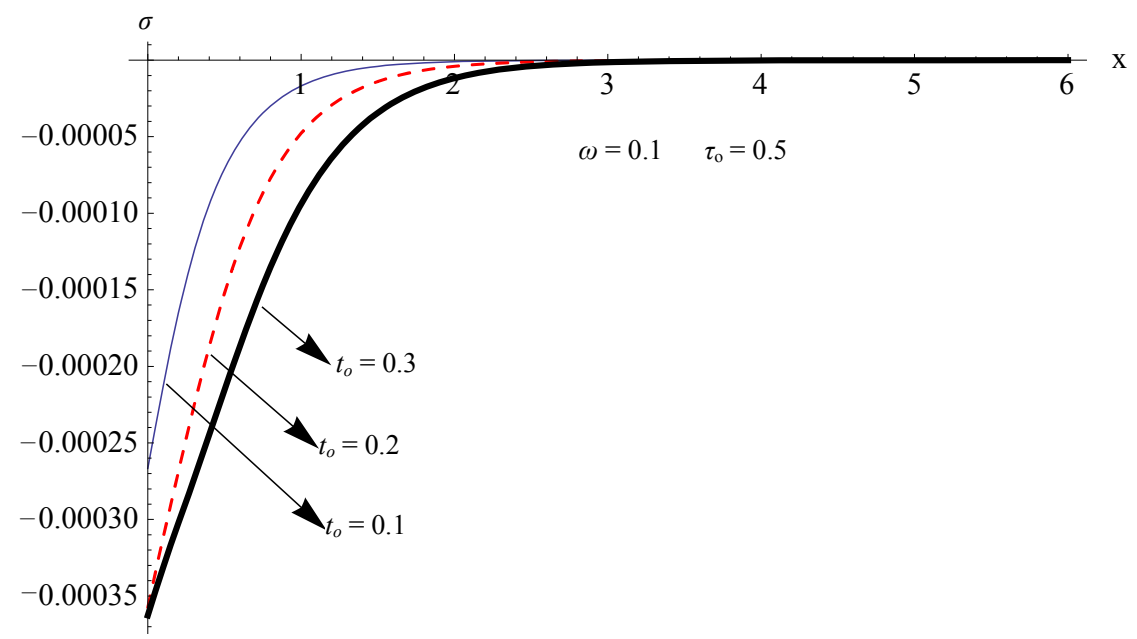

Figure 26. The stress distribution at different time in the presence of two temperature parameter at $\tau_{o}=0.5$. 


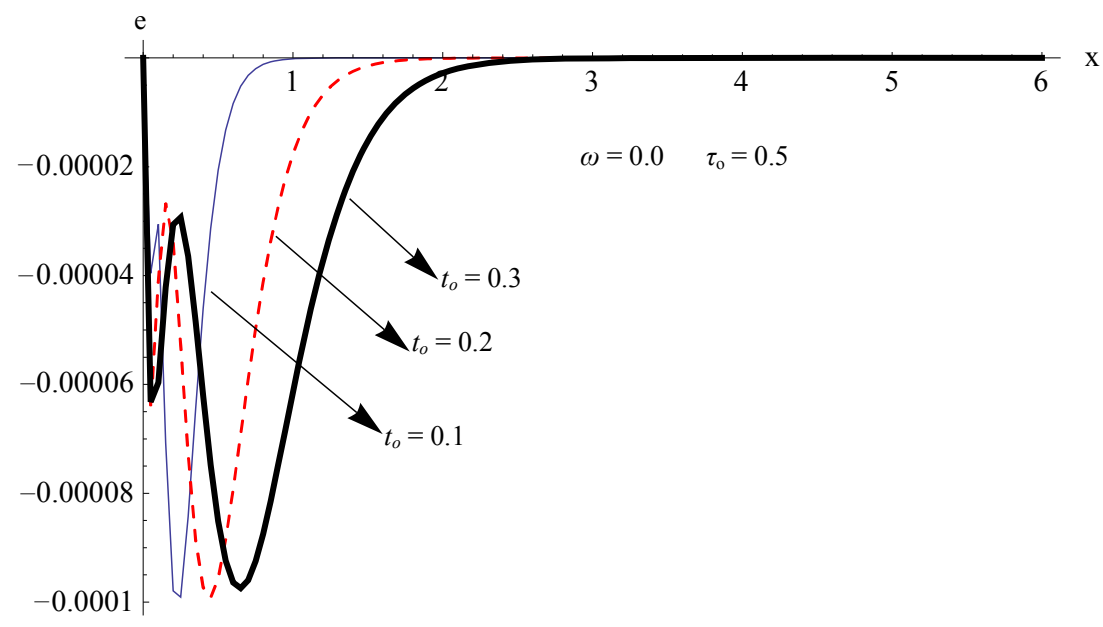

Figure 27. The strain distribution at different time in the absent of two temperature parameter $\tau_{o}=0.5$.

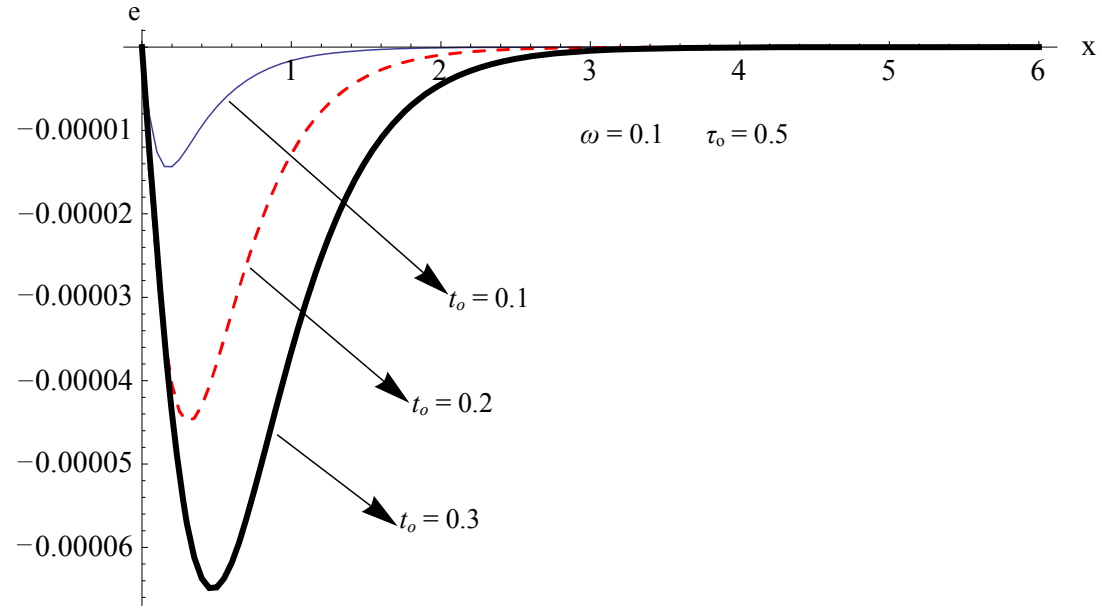

Figure 28. The strain distribution at different time in the presence of two temperature parameter at $\tau_{o}=0.5$.

University at Alkharj-KSA, as this work has carried out according to the contract: $23 / \mathrm{h} / 1432$.

\section{REFERENCES}

[1] J. Tani, T. Takagi and J. Qiu, "Intelligent Material Systems: Application of Functional Materials," Applied Mechanics Review, Vol. 51, No. 8, 1998, pp. 505-521. doi:10.1115/1.3099019

[2] H. S. Tzou, "Multifield Transducers, Devices, Mechatronics Systems and Structronic Systems with Smart Materials," The Shock and Vibration Digest, Vol. 30, No. 4, 1998, pp. 282-294. doi:10.1177/058310249803000402

[3] S. S. Rao and M. Sunar, "Piezoelectricity and Its Use in Disturbance Sensing and Control of Flexible Structures: A Survey," Applied Mechanics Review, Vol. 47, No. 4, 1994, pp. 113-123. doi:10.1115/1.3111074

[4] H. W. Lord and Y. Shulman, "A Generalized Dynamical Theory of Thermoelasticity," Journal of the Mechanics and Physics of Solids, Vol. 15, No. 5, 1967, pp. 299-309. doi:10.1016/0022-5096(67)90024-5

[5] A. E. Green and K. E. Lindsay, "Thermoelasticity," Journal of Elasticity, Vol. 2, No. 1, 1972, pp. 1-7. doi:10.1007/BF00045689

[6] E. Bassiouny and H. Youssef, "Two-Temperature Generalized Thermopiezoelasticity of Finite Rod Subjected to Different Types of Thermal Loading," Journal of Thermal Stresses, Vol. 31, No. 3, 2008, pp. 233-245. doi:10.1080/01495730701737902

[7] T. H. He, X. G. Tian and Y. P. Shen, "State Space Approach to One-Dimensional Shock Problem for a SemiInfinite Piezoelectric Rod," International Journal of Engineering Science, Vol. 40, No. 10, 2002, pp. 1081-1097. doi:10.1016/S0020-7225(02)00005-8

[8] G. Hanig and U. Hirdes, "A Method for the Numerical Inversion of Laplace Transform," Journal of Computational and Applied Mathematics, Vol. 10, No. 1, 1984, pp. 113-132. doi:10.1016/0377-0427(84)90075-X 


\section{Nomenclature}

$A_{i j} \quad$ The components of relaxation time.

$a$ The two-temperature parameter.

$C_{E} \quad$ Specific heat at constant strain.

$c_{i j k l} \quad$ The elastic constants.

$c_{o}=\sqrt{\frac{\lambda+2 \mu}{\rho}}$ Longitudinal wave speed.

$D_{i} \quad$ The components of electric displacement.

$d_{i} \quad$ The pyroelectric constants.

$E_{i} \quad$ The components of electric field vector.

$e_{i j k l} \quad$ The components of strain tensor.

$h_{i j k} \quad$ The piezoelectric coefficients.

$k_{i j} \quad$ The components of thermal conductivity.

$q_{i} \quad$ The components of the heat flux vector.

$T \quad$ Absolute temperature.

$T_{o} \quad$ Reference temperature.

$t$ Time.

$t_{o} \quad$ Ramping time parameter.

$u_{i} \quad$ Components of displacement vector.

$v_{i} \quad$ The electric potential function.

$\alpha=\frac{\gamma T_{o}}{\lambda+2 \mu}$ Dimensionless thermoelastic coupling constant.
$\alpha_{T} \quad$ Coefficient of linear thermal expansion.

$\tau_{i k} \quad$ The components of dielectric tensor.

$\beta_{i j} \quad$ The thermal modulus.

$\gamma=(3 \lambda+2 \mu) \alpha_{T}$.

$\Omega \quad$ The angular frequency of thermal vibration.

$\delta_{i j} \quad$ Kronecker delta function.

$\varepsilon=\frac{\gamma}{\rho C_{E}}$ Dimensionless mechanical coupling constant.

$\zeta \quad$ The entropy density.

$\eta=\frac{\rho C_{E}}{k}$ The thermal viscosity.

$C=\frac{\rho C_{E}}{T_{o}}$

$\theta=\left(T-T_{o}\right)$ The dynamical temperature increment such

that $\frac{\left|T-T_{o}\right|}{T_{o}} \ll 1$.

$\lambda, \mu \quad$ Lamé's constants.

$\rho$ Mass density.

$\sigma_{i j} \quad$ Components of stress tensor.

$\sigma=\sigma_{x x}$ The principal stress component.

$\tau_{o} \quad$ One relaxation time parameter. 